\title{
Treatment of hypopharyngeal carcinoma complicated with diabetic nephropathy: a case report and literature review
}

\author{
Wei Liu ${ }^{1,2 \#}$, Jin-Fu Peng ${ }^{2 \#}$, Meng-Jie Tang ${ }^{1}$ \\ ${ }^{1}$ Department of Pathology, Hunan Cancer Hospital/The Affiliated Cancer Hospital of Xiangya School of Medicine, ${ }^{2}$ Department of Pharmacy, The \\ Third Xiangya Hospital, Central South University, Changsha 410013, China \\ \#These authors contributed equally to this work. \\ Correspondence to: Meng-Jie Tang. Hunan Cancer Hospital/The Affiliated Cancer Hospital of Xiangya School of Medicine, Central South University, \\ Changsha 410013, China. Email: tangmengjie@hnca.org.cn.
}

\begin{abstract}
For elderly cancer patients with diabetic nephropathy, severe renal insufficiency leads to a very high risk of chemotherapy. Physicians and pharmacists must consider both the benefits of cancer treatment and the impact of antineoplastic drugs on patients' residual renal function, and choose appropriate chemotherapy regimens to balance the efficacy and safety of drugs. We report a case of a patient who presented with dysphagia and sore throat with serum creatinine of $169 \mu \mathrm{mol} / \mathrm{L}$ and fasting blood glucose of $7.9 \mathrm{mmol} / \mathrm{L}$ on admission. The main diagnosis was hypopharyngeal carcinoma with diabetic nephropathy. The clinical pharmacist reviewed the literature and analyzed the pharmacological and pharmacokinetic characteristics of anti-tumor drugs in patients, and adjusted the chemotherapy regimen and dose according to the renal function of patients. To the best of our knowledge, this is the first reported case of hypopharyngeal carcinoma with severe renal insufficiency treated successfully with multimodality therapy in China. The purpose of this case is to optimize the anti-tumor treatment regimen and drug dose adjustment of hypopharyngeal carcinoma with severe renal insufficiency, so as to provide a reference for clinicians and clinical pharmacists to use drugs rationally.
\end{abstract}

Keywords: Hypopharyngeal carcinoma; diabetic nephropathy; renal insufficiency; therapeutic regimen; dose adjustment; case report

Submitted Nov 21, 2019. Accepted for publication Dec 12, 2019.

doi: $10.21037 /$ tcr.2019.12.42

View this article at: http://dx.doi.org/10.21037/tcr.2019.12.42

\section{Introduction}

The hypopharynx is located posterior and inferior to the oropharynx and extends to the esophageal inlet. Hypopharyngeal malignancies account for $6 \%$ of head and neck malignancies (1), and more than $95 \%$ of hypopharyngeal cancers are squamous cell carcinomas (2). At the time of diagnosis, less than $15 \%$ of hypopharyngeal cancers are confined to the hypopharynx and most have spread to regional lymph nodes or distally. The presence of albuminuria was considered to be an early sign of classical diabetic glomerulopathy, which is characterized by glomerular basement membrane thickening, endothelial damage, mesangial expansion and nodules, and podocytes loss (3). It occurs most frequently in patients with type 1 and type 2 diabetes who have a long duration of disease and poor glycemic control, and hypertension, glomerular hyperfiltration rate, and genetic susceptibility are also highrisk factors for its development (4).

Diabetic nephropathy is one of the main causes of endstage renal disease. For elderly cancer patients with diabetic nephropathy, severe renal insufficiency leads to a very high risk of chemotherapy. Physicians and pharmacists must consider both the benefits of cancer treatment and the impact of antineoplastic drugs on patients' residual renal function, and choose appropriate chemotherapy regimens to balance the efficacy and safety of drugs. This article discusses the course of treatment of a patient with 


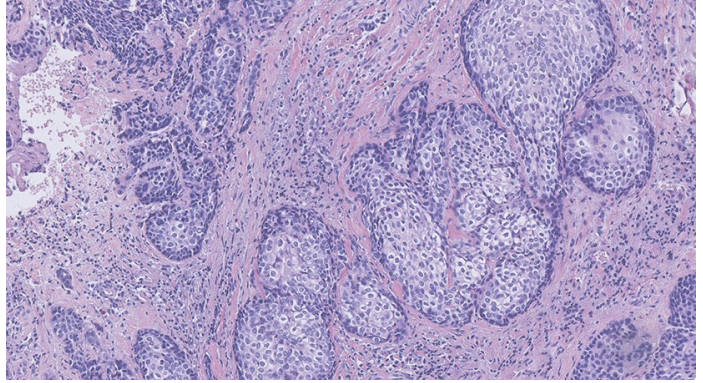

Figure 1 Analysis of primary hypopharyngeal lesion tissue. Hematoxylin and eosin staining of the hypopharyngeal lesion tissue (20× magnification).

hypopharyngeal carcinoma and diabetic nephropathy diagnosed with severe renal insufficiency. We analyzed the antineoplastic regimen and drug dosage in this patient based on a literature review to improve the treatment strategy for such patients.

\section{Case presentation}

A 75 -year-old Chinese man presented in June 2018 with dysphagia, sore throat, cracked piriform fossa and left lateral wall of oropharynx with superficial ulceration on electronic laryngoscope, rough left laryngopharyngeal mucosa, and swelling in the interarytenoid region. Computed tomography (CT) showed lesions involving the upper esophagus, involving the left sternocleidomastoid muscle, multiple enlarged lymph nodes around the mass and in the neck, and enlarged retropharyngeal and parapharyngeal lymph nodes. The diagnosis of hypopharyngeal carcinoma (moderately differentiated squamous cell carcinoma) was confirmed after perfecting biopsy pathological examination (Figure 1). The TNM stage of the tumor was $\mathrm{cT} 4 \mathrm{aN} 2 \mathrm{bM} 0$, stage IVA. The patient had diabetes for 20 years and diabetic nephropathy for 7 years with a glomerular filtration rate (GFR) of $28.86 \mathrm{~mL} / \mathrm{min}$. The patient had a 40 -year history of smoking and alcohol consumption, was married and fertile, had a healthy family, and denied exposure to the affected area and a history of associated familial genetic disease. Based on the patient's tumor pathological characteristics, prognostic factors, age, and patient's performance status, TCF regimen induction chemotherapy was started for 3 cycles in July 2018: docetaxel $75 \mathrm{mg} / \mathrm{m}^{2}$, carboplatin 270-334 mg, and 5-Fluorouracil (5-FU) $750 \mathrm{mg} / \mathrm{m}^{2}$ by continuous infusion in a micro-pump for 5 days in every 21 -day cycle. The patient was treated radically with helical TomoTherapy in October 2018. The patient tolerated the treatment well and no serious toxicity issues were noted. At present, the patient had no recurrence of disease.

\section{Discussion}

\section{Hypopharyngeal carcinoma treatment regimens}

Treatments for hypopharyngeal cancer include surgery, radiation therapy, concurrent chemoradiotherapy, induction chemotherapy, and biological therapy (5). Because the site of the disease is hidden and there are few early cases, $80 \%$ of patients already have stage III and IV disease at presentation. It is difficult to treat advanced lesions of hypopharyngeal carcinoma. When the local tumors are large (T3 and T4 lesions), more than $60 \%$ of them have submucosal extension of the tumor. Because it is difficult to ensure its safe margin by surgery, it is easy to cause residual tumor. Although surgical treatment can completely remove the tumor, due to a wide range of resection, especially circumferential defects, it is often difficult to repair or because the trauma is too general for the patient to tolerate, easily resulting in some serious complications (6). According to The National Comprehensive Cancer Network (NCCN) Clinical Practice Guidelines: Head and Neck Cancer (2018. V1): for most well-behaved patients with locally advanced (stage III or IV) laryngeal or hypopharyngeal cancer, a functional organ preservation strategy rather than surgical resection is recommended (grade 1B). Organ preservation methods can allow preservation of the larynx but offer no survival advantage over total laryngectomy. Concurrent chemoradiotherapy, induction chemotherapy, radiation therapy alone, and sequential therapy are all used as functional organ preservation techniques. Postoperative radiotherapy or concurrent chemoradiotherapy can improve the survival rate of patients in the high-risk group; preoperative induction chemotherapy, surgery or radiotherapy selected according to the results of induction chemotherapy, radiotherapy for those who are sensitive to induction chemotherapy, and surgery for those who are not sensitive, the long-term survival rate results are similar, and induction chemotherapy can make the treatment choice of patients more individualized. For patients with N0, N1, and $\mathrm{N} 2 \mathrm{a}$ manifestations, concurrent chemoradiotherapy is preferred; whereas induction chemotherapy can provide a survival advantage for patients with a relatively high risk of distant metastasis (large N2a, N2b, N2c, and N3) (7). The 
patient was diagnosed with hypopharyngeal malignancy (squamous cell carcinoma, cT4aN2bM0, stage IVA, ECOG score 1) and met the guidelines and the recommendations of the literature selection for induction chemotherapy.

The regimen of induction chemotherapy can be the traditional cisplatin and 5-FU (PF) regimen (8), but recent studies have shown that TPF regimen added to docetaxel is superior to PF regimen (Class 1 evidence) (9). For advanced hypopharyngeal cancer, 2-3 cycles of induction chemotherapy are generally applied. Complete remission was achieved by evaluation after induction chemotherapy, and radical radiotherapy was performed; if most regression was achieved, the next treatment was surgery, or concurrent chemoradiotherapy; if the lesion did not shrink, radical surgery was performed, and adjuvant radiotherapy or chemoradiotherapy was performed according to pathological results after surgery. The guideline recommended dosing regimen is: docetaxel $75 \mathrm{mg} / \mathrm{m}^{2}$ day 1 , cisplatin $75 \mathrm{mg} / \mathrm{m}^{2}$ day 1 , and $5-\mathrm{FU}$ $750 \mathrm{mg} / \mathrm{m}^{2}$ by continuous infusion in a micro-pump for 5 days in every 21-day cycle (7).

\section{Adjustment of chemotherapy regimen and drug dose}

Serum drug concentrations are not usually measured for chemotherapeutic agents, and dose adjustment in this setting is usually based on 5-FU and renal toxicity of the agent. Docetaxel is primarily metabolized by the liver with less than $5 \%$ renal excretion, and patients with renal insufficiency have little effect on (pharmacokinetic) PK parameters and no dose adjustment is necessary (10). Cisplatin is one of the largest agents of nephrotoxicity and may cause acute kidney injury, thrombotic microangiopathy, hypomagnesemia, proximal tubular dysfunction, and anemia disproportionate to the myelosuppressive effects of the drug. The use of cisplatin in patients with underlying renal insufficiency and elevated serum creatinine levels is associated with an increased risk of acute kidney injury, but there are currently no definitive studies to help guide the threshold level of renal function at which cisplatin is contraindicated. The US Prescribing Information recommends that cisplatin should be avoided in patients with serum creatinine $>1.5 \mathrm{mg} / \mathrm{dL}$ or an estimated GFR $<50 \mathrm{~mL} / \mathrm{min}$. Although a few studies have indicated that cisplatin can be given in reduced doses to patients with renal insufficiency, substantial data validation is lacking to support their recommendations, and their specific suggested dose adjustment regimens are not consistent (11).
Replacement of cisplatin with carboplatin may be considered for patients with hypopharyngeal cancer in whom cisplatin is contraindicated or in whom the risk of nephrotoxicity is increased (12). In patients with advanced squamous cell carcinoma of the head and neck, carboplatin combined with taxanes is also a very effective chemotherapy regimen when cisplatin is not feasible (13). Carboplatin is significantly less nephrotoxic than cisplatin (14). The importance of renal clearance for carboplatin metabolism and excretion is emphasized by its usual dose regimen. This regimen was calculated based on estimated GFR and area under the concentration-time curve (AUC) rather than based on body surface area (BSA), using the Calvert formula: total carboplatin dose $(\mathrm{mg})=$ target $\mathrm{AUC} \times(\mathrm{GFR}+25)$; it usually varies between 5 and 7 using the desired target AUC (15). Due to potential changes in body weight or renal function, this should be recalculated prior to each dose of carboplatin chemotherapy. In clinical practice, actual body weight is generally used to estimate GFR by the Cockcroft-Gault formula to calculate the carboplatin dose; however, the use of actual body weight in obese patients can result in overestimation of GFR and overestimation of carboplatin dose (16). The optimal body weight regarding the Calvert formula for calculating the carboplatin dose is not addressed in the American Society of Clinical Oncology (ASCO) guidelines. The Gynecologic Oncology Group (GOG) recommends that actual body weight be used to estimate GFR as long as the patient's body mass index (BMI) is $<25 \mathrm{~kg} / \mathrm{m}^{2}$ when using the Cockcroft-Gault formula, and for patients with $B M I \geq 25 \mathrm{~kg} / \mathrm{m}^{2}$, corrected body weight $\{[($ actual body weight - ideal body weight $) \times 0.4]+$ ideal body weight is recommended (17). Not all regimens containing carboplatin use AUC-based dosing, and the US prescribing information for carboplatin indicates the following renalfunction-based dose reduction regimens: initial dose reduction from $300-360$ to $250 \mathrm{mg} / \mathrm{m}^{2}$ at GFR $41-$ $59 \mathrm{~mL} / \mathrm{min}$, initial dose reduction to $200 \mathrm{mg} / \mathrm{m}^{2}$ at GFR $16-40 \mathrm{~mL} / \mathrm{min}$, and no recommendation for use at GFR $\leq 15 \mathrm{~mL} / \mathrm{min}$. 5 -FU is not cleared by renal function mechanisms, so no dose adjustment is required in patients with renal insufficiency (10).

This patient had a GFR of $28.86 \mathrm{~mL} / \mathrm{min}$ and a BSA of $1.67 \mathrm{~m}^{2}$ and was clinically diagnosed with severe renal insufficiency and chronic kidney disease (CKD) stage 4. Taking into account the results of the above studies as well as the specific patient conditions, we performed a chemotherapy regimen and dose adjustment: the TCF regimen of carboplatin plus docetaxel and fluorouracil was 
Table 1 Biochemical and routine indicators of peripheral blood in patients

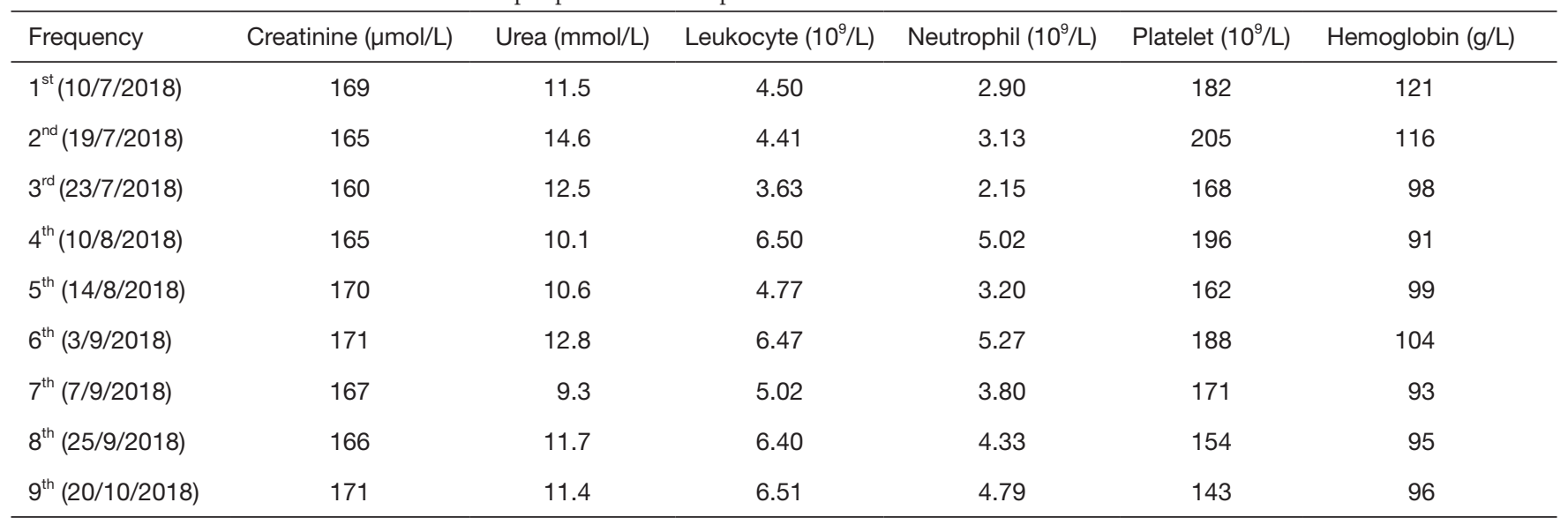

used instead of the TPF regimen of cisplatin plus docetaxel and fluorouracil. The dose of carboplatin was adjusted from the standard dose to $270-334 \mathrm{mg}$ of theoretical dose, and the median dose was $300 \mathrm{mg}$; no dose adjustment was required for docetaxel and $5-\mathrm{FU}$.

\section{Safety and effectiveness}

The patient tolerated the treatment well. Leukopenia, neutropenia, and thrombocytopenia did not occur; no clinically assessed neurotoxicity was observed; and chemotherapy induced Grade I-II anemia (CTCAE). During the whole chemotherapy and follow-up, the serum creatinine and urea values of the patients showed no significant change compared with those before chemotherapy, indicating that the TCF regimen of carboplatin plus docetaxel and fluorouracil in this study had no significant effect on the renal function of patients (Table 1). After completing 3 cycles of induction chemotherapy, the patient underwent neck CT. The results suggested that the range of lesions was shrinking than before, the disease condition was improved than before, the efficacy evaluation criteria for partial remission of tumor disease were met, and the purpose of induction chemotherapy was achieved. After radical radiotherapy, the patient was in good general condition during the follow-up period, and no relevant evidence of disease progression or recurrence was found.

What is special about this study is that patients with hypopharyngeal cancer and severe renal insufficiency have completed standard treatment and achieved satisfactory clinical outcomes through individualized adjustment of chemotherapy regimens and drug doses. A limitation of this study is that the success of a single patient is difficult to guide a broad clinical extension, and in the future, we also need to conduct a large sample of randomized controlled trial studies to further validate our results. In conclusion, severe renal insufficiency is not a contraindication for the treatment of hypopharyngeal cancer. Improved TCF regimen of carboplatin plus docetaxel and fluorouracil may be a safe and effective chemotherapy regimen for patients with hypopharyngeal cancer and severe renal insufficiency. Through multidisciplinary cooperation, selection of a reasonable individualized treatment plan, and close monitoring of renal function, patients with hypopharyngeal cancer with severe renal insufficiency can still complete standard treatment.

\section{Acknowledgments}

Funding: This study was supported by youth fund of National Natural Science Foundation of China (Grant No. 81603192).

\section{Footnote}

Conflicts of Interest: All authors have completed the ICMJE uniform disclosure form (available at http://dx.doi. org/10.21037/tcr.2019.12.42). The authors have no conflicts of interest to declare.

Ethical Statement: The authors are accountable for all aspects of the work in ensuring that questions related to the accuracy or integrity of any part of the work are 
appropriately investigated and resolved. All procedures performed in studies involving human participants were in accordance with the Declaration of Helsinki (as revised in 2013). Written informed consent for publication of this case report was obtained from the patient.

Open Access Statement: This is an Open Access article distributed in accordance with the Creative Commons Attribution-NonCommercial-NoDerivs 4.0 International License (CC BY-NC-ND 4.0), which permits the noncommercial replication and distribution of the article with the strict proviso that no changes or edits are made and the original work is properly cited (including links to both the formal publication through the relevant DOI and the license). See: https://creativecommons.org/licenses/by-nc-nd/4.0/.

\section{References}

1. Carvalho AL, Nishimoto IN, Califano JA, et al. Trends in incidence and prognosis for head and neck cancer in the United States: a site-specific analysis of the SEER database. Int J Cancer 2005;114:806-16.

2. Patel RS, Goldstein DP, Brown D, et al. Circumferential pharyngeal reconstruction: history, critical analysis of techniques, and current therapeutic recommendations. Head Neck 2010;32:109-20.

3. Chapter 1: Definition and classification of CKD. Kidney Int Suppl (2011) 2013;3:19-62.

4. Ritz E, Orth SR. Nephropathy in patients with type 2 diabetes mellitus. N Engl J Med 1999;341:1127-33.

5. Martin A, Jackel MC, Christiansen H, et al. Organ preserving transoral laser microsurgery for cancer of the hypopharynx. Laryngoscope 2008;118:398-402 .

6. Mura F, Bertino G, Occhini A, et al. Surgical treatment of hypopharyngeal cancer: a review of the literature and proposal for a decisional flow-chart. Acta Otorhinolaryngol Ital 2013;33:299-306.

7. Colevas AD, Yom SS, Pfister DG, et al. NCCN Guidelines
Insights: Head and Neck Cancers, Version 1.2018. J Natl Compr Canc Netw 2018;16:479-90.

8. Blanchard P, Baujat B, Holostenco V, et al. Meta-analysis of chemotherapy in head and neck cancer (MACH-NC): a comprehensive analysis by tumour site. Radiother Oncol 2011;100:33-40.

9. Vermorken JB, Remenar E, van Herpen C, et al. Cisplatin, fluorouracil, and docetaxel in unresectable head and neck cancer. N Engl J Med 2007;357:1695-704.

10. Aronoff G. Drug prescribing in renal failure. 5th edition. Philadelphia, PA: ACP Press, 2007.

11. Kintzel PE, Dorr RT. Anticancer drug renal toxicity and elimination: dosing guidelines for altered renal function. Cancer Treat Rev 1995;21:33-64.

12. Posner MR, Norris CM, Wirth LJ, et al. Sequential therapy for the locally advanced larynx and hypopharynx cancer subgroup in TAX 324: survival, surgery, and organ preservation. Ann Oncol 2009;20:921-7.

13. Haddad R, Sonis S, Posner M, et al. Randomized phase 2 study of concomitant chemoradiotherapy using weekly carboplatin/paclitaxel with or without daily subcutaneous amifostine in patients with locally advanced head and neck cancer. Cancer 2009;115:4514-23.

14. McDonald BR, Kirmani S, Vasquez M, et al. Acute renal failure associated with the use of intraperitoneal carboplatin: a report of two cases and review of the literature. Am J Med 1991;90:386-91.

15. Calvert AH, Newell DR, Gumbrell LA, et al. Carboplatin dosage: prospective evaluation of a simple formula based on renal function. J Clin Oncol 1989;7:1748-56.

16. De Jonge ME, Mathot RA, Van Dam SM, et al. Extremely high exposures in an obese patient receiving high-dose cyclophosphamide, thiotepa and carboplatin. Cancer Chemother Pharmacol 2002;50:251-5.

17. Ekhart C, Rodenhuis S, Schellens JH, et al. Carboplatin dosing in overweight and obese patients with normal renal function, does weight matter? Cancer Chemother Pharmacol 2009;64:115-22.
Cite this article as: Liu W, Peng JF, Tang MJ. Treatment of hypopharyngeal carcinoma complicated with diabetic nephropathy: a case report and literature review. Transl Cancer Res 2020;9(3):2040-2044. doi: 10.21037/tcr.2019.12.42 
Supplementary

Table S1 Historical and current information from this episode of care organized as a timeline

\begin{tabular}{ll}
\hline Date & Care \\
\hline $5 / 7 / 2018$ & Electronic laryngoscopy + laryngoscope biopsy of hypopharyngeal mass \\
$13 / 7 / 2018$ & Neck CT + chest CT \\
$21 / 7 / 2018$ & Chemotherapy (docetaxel $75 \mathrm{mg} / \mathrm{m}^{2}$, carboplatin $300 \mathrm{mg}$, and $5-\mathrm{FU} 750 \mathrm{mg} / \mathrm{m}^{2}$ ) \\
$12 / 8 / 2018$ & Chemotherapy (docetaxel $75 \mathrm{mg} / \mathrm{m}^{2}$, carboplatin $300 \mathrm{mg}$, and $5-\mathrm{FU} 750 \mathrm{mg} / \mathrm{m}^{2}$ ) \\
$4 / 9 / 2018$ & Chemotherapy (docetaxel $75 \mathrm{mg} / \mathrm{m}^{2}$, carboplatin $300 \mathrm{mg}$, and $5-\mathrm{FU} 750 \mathrm{mg} / \mathrm{m}^{2}$ ) \\
$21 / 10 / 18$ & Radical radiation therapy \\
\hline
\end{tabular}

